\title{
The Effect of Tensor Interaction in Splitting the Energy Levels of Relativistic Systems
}

\author{
Mohammad Reza Shojaei and Mohsen Mousavi \\ Department of Physics, University of Shahrood, P.O. Box 36155-316, Shahrood, Iran \\ Correspondence should be addressed to Mohsen Mousavi; nuclear.physics2020@gmail.com
}

Received 9 September 2015; Revised 10 November 2015; Accepted 22 November 2015

Academic Editor: Sally Seidel

Copyright ( 2016 M. R. Shojaei and M. Mousavi. This is an open access article distributed under the Creative Commons Attribution License, which permits unrestricted use, distribution, and reproduction in any medium, provided the original work is properly cited. The publication of this article was funded by SCOAP ${ }^{3}$.

\begin{abstract}
We solve approximately Dirac equation for Eckart plus Hulthen potentials with Coulomb-like and Yukawa-like tensor interaction in the presence of spin and pseudospin symmetry for $k \neq 0$. The formula method is used to obtain the energy eigenvalues and wave functions. We also discuss the energy eigenvalues and the Dirac spinors for Eckart plus Hulthen potentials with formula method. To show the accuracy of the present model, some numerical results are shown in both pseudospin and spin symmetry limits.
\end{abstract}

\section{Introduction}

One of the interesting problems in nuclear and high energy physics is to obtain analytical solution of the Klein-Gordon, Duffin-Kemmer-Petiau, and Dirac equations for mixed vector and scalar potentials [1]. The study of relativistic effect is always useful in some quantum mechanical systems $[2,3]$. Therefore, the Dirac equation has become the most appealing relativistic wave equation for spin-1/2 particles. For example, in the relativistic treatment of nuclear phenomena, the Dirac equation is used to describe the behavior of the nuclei in nucleus and also to solve many problems of high energy physics and chemistry. For this reason, it has been used extensively to study the relativistic heavy ion collisions and heavy ion spectroscopy and more recently in laser-matter interaction (for a review, see [4] and references therein) and condensed matter physics $[5,6]$. The idea about spin symmetry and pseudospin symmetry with the nuclear shell model has been introduced in 1969 by Arima et al. (1969) and Hecht and Adler (1969) [7, 8]. Spin and pseudospin symmetries are $\mathrm{SU}(2)$ symmetries of a Dirac Hamiltonian with vector and scalar potentials. They are realized when the difference, $\Delta(r)=V(r)-S(r)$, or the sum, $\sum(r)=V(r)+$ $S(r)$, is constant. The near realization of these symmetries may explain degeneracy in some heavy meson spectra (spin symmetry) or in single-particle energy levels in nuclei (pseudospin symmetry), when these physical systems are described by relativistic mean-field theories (RMF) with scalar and vector potentials [9]. Recently, some authors have studied various types of potential with a tensor potential, under the conditions of pseudospin and spin symmetry. They have found out that the tensor interaction removes the degeneracy between two states in the pseudospin and spin doublet [1013]. The pseudospin and spin symmetry appearing in nuclear physics refers to a quasidegeneracy of the single-nucleon doublets and can be characterized with the nonrelativistic quantum numbers $(n, l, j=l+1 / 2)$ and $(n, l+2, j=$ $l+3 / 2$ ), where $n, l$, and $j$ are the single-nucleon radial, orbital, and total angular momentum quantum numbers for a single particle, respectively $[7,8]$. These kinds of various methods have been used for the analytical solutions of the Klein-Gordon equation and Dirac equation such as the super symmetric quantum mechanics [14-16], asymptotic iteration method (AIM) [17, 18], factorization method [19, 20], Laplace transform approach [21], GPS method [22, 23] and the path integral method [24-26], and Nikiforov-Uvarov method [2729]. The Klein-Gordon and Dirac wave equations are frequently used to describe the particle dynamics in relativistic quantum mechanics with some typical kinds of potential by using different methods [30]. For example, Kratzer potential 
$[31,32]$, Woods-Saxon potential $[33,34]$, Scarf potential [35, 36], Hartmann potential [37, 38], Rosen Morse potential, $[39,40]$, Hulthen potential [41], and Eckart potential [42, 43].

In this paper, we attempt to solve approximately Dirac wave equation for $k \neq 0$ for Eckart plus Hulthen potentials for the spin and pseudospin symmetry with a tensor potential by using the formula method. The organization of this paper is as follows: in Section 2, the formula method is reviewed [44]. In Section 3 we review basic Dirac equations briefly. In Sections 3.1 and 3.2, solutions of Dirac wave equation for the spin and pseudospin symmetry of these potentials in the presence of Coulomb-like tensor interaction are presented, respectively. In Sections 3.3 and 3.4, solutions of Dirac wave equation for the spin and pseudospin symmetry of these potentials in the presence of Coulomb-like plus Yukawa-like tensor interaction are presented, respectively. In Section 4, we provide results and discussion. The conclusion is given in Section 5 .

\section{Review of Formula Method}

The formula method has been used to solve the Schrodinger, Dirac, and Klein-Gordon wave equations for a certain kind of potential. In this method, the differential equations can be written as follows [44]:

$$
\begin{aligned}
& \Psi_{n}^{\prime \prime}(s)+\frac{\left(k_{1}-k_{2} s\right)}{s\left(1-k_{3} s\right)} \Psi_{n}^{\prime}(s)+\frac{\left(A s^{2}+B s+C\right)}{s^{2}\left(1-k_{3} s\right)^{2}} \Psi_{n}(s) \\
& \quad=0 .
\end{aligned}
$$

For a given Schrödinger-like equation in the presence of any potential model which can be written in the form of (1), the energy eigenvalues and the corresponding wave function can be obtained by using the following formulas, respectively [44]:

$$
\begin{aligned}
& {\left[\frac{k_{4}^{2}-k_{5}^{2}-\left[((1-2 n) / 2)-\left(1 / 2 k_{3}\right)\left(k_{2}-\sqrt{\left(k_{3}-k_{2}\right)^{2}-4 A}\right)\right]^{2}}{2\left[((1-2 n) / 2)-\left(1 / 2 k_{3}\right)\left(k_{2}-\sqrt{\left(k_{3}-k_{2}\right)^{2}-4 A}\right)\right]}\right]^{2}-k_{5}^{2}=0, \quad k_{3} \neq 0,} \\
& \Psi_{n}(s)=N_{n} s^{k_{4}}\left(1-k_{3} s\right)^{k_{5}}{ }_{2} F_{1}\left(-n, n+2\left(k_{4}+k_{5}\right)+\frac{k_{2}}{k_{3}}-1 ; 2 k_{4}+k_{1}, k_{3} s\right),
\end{aligned}
$$

where

$$
\begin{aligned}
k_{4}= & \frac{\left(1-k_{1}\right)+\sqrt{\left(1-k_{1}\right)^{2}-4 C}}{2}, \\
k_{5}= & \frac{1}{2}+\frac{k_{1}}{2}-\frac{k_{2}}{2 k_{3}} \\
& +\sqrt{\left[\frac{1}{2}+\frac{k_{1}}{2}-\frac{k_{2}}{2 k_{3}}\right]^{2}-\left[\frac{A}{k_{3}^{2}}+\frac{B}{k_{3}}+C\right]} .
\end{aligned}
$$

And $N_{n}$ is the normalization constant. In special case where $k_{3} \rightarrow 0$, the energy eigenvalues and the corresponding wave function can be obtained as [44]

$$
\begin{aligned}
& {\left[\frac{B-k_{4} k_{2}-n k_{2}}{2 k_{4}+k_{1}+2 n}\right]^{2}-k_{5}^{2}=0,} \\
& \Psi_{n}(s) \\
& \quad=N_{n} s^{k_{4}} \exp \left(-k_{5} s\right)_{1} F_{1}\left(-n ; 2 k_{4}+k_{1} ;\left(2 k_{5}+k_{2}\right) s\right) .
\end{aligned}
$$

The solution provides a valuable means for checking and improving models and numerical methods introduced for solving complicated quantum systems.

\section{Basic Dirac Equations}

In the relativistic description, the Dirac equation of a single nucleon with the mass moving in an attractive scalar potential
$S(r)$ and a repulsive vector potential $V(r)$ can be written as [45]

$$
\left[-i \hbar c \hat{\alpha} \cdot \widehat{\nabla}+\widehat{\beta}\left(M c^{2}+S(r)\right)\right] \Psi_{n_{r}, k}=[E-V(r)] \Psi_{n_{r}, k},
$$

where $E$ is the relativistic energy, $M$ is the mass of a single particle, and $\alpha$ and $\beta$ are the $4 \times 4$ Dirac matrices. For a particle in a central field, the total angular momentum $J$ and $\widehat{K}=-\widehat{\beta}(\widehat{\alpha} \cdot \widehat{L}+\hbar)$ commute with the Dirac Hamiltonian, where $L$ is the orbital angular momentum. For a given total angular momentum $j$, the eigenvalues of the $\widehat{K}$ are $k=$ $\pm(j+1 / 2)$, where negative sign is for aligned spin and positive sign is for unaligned spin. The wave functions can be classified according to their angular momentum $j$ and spinorbit quantum number $k$ as follows:

$$
\Psi_{n_{r}, k}(r, \theta, \phi)=\frac{1}{r}\left[\begin{array}{c}
F_{n_{r}, k}(r) Y_{j m}^{l}(\theta, \phi) \\
i G_{n_{r}, k}(r) Y_{j m}^{\widetilde{l}}(\theta, \phi)
\end{array}\right],
$$

where $F_{n_{r}, k}(r)$ and $G_{n_{r}, k}(r)$ are upper and lower components and $Y_{j m}^{l}(\theta, \phi)$ and $Y_{j m}^{\tilde{l}}(\theta, \phi)$ are the spherical harmonic functions. $n_{r}$ is the radial quantum number and $m$ is the projection of the angular momentum on the $z$-axis. The orbital angular momentum quantum numbers $l$ and $\widetilde{l}$ represent the spin and pseudospin quantum numbers. Substituting (7) into (6), 
we obtain couple equations for the radial part of the Dirac equation as follows by $\hbar=c=1$ :

$$
\begin{aligned}
& \left(\frac{d}{d r}+\frac{k}{r}-U(r)\right) F_{n_{r}, k}(r) \\
& =\left[M+E_{n, k}-\Delta(r)\right] G_{n_{r}, k}(r), \\
& \left(\frac{d}{d r}-\frac{k}{r}+U(r)\right) G_{n_{r}, k}(r) \\
& \quad=\left[M-E_{n, k}+\sum(r)\right] F_{n_{r}, k}(r),
\end{aligned}
$$

where $\Delta(r)=V(r)-S(r)$ and $\sum(r)=V(r)+S(r)$ are the difference and the sum of the potentials $V(r)$ and $S(r)$, respectively, and $U(r)$ is a tensor potential. We obtain the second-order Schrodinger-like equation as

$$
\begin{aligned}
& \left\{\frac{d^{2}}{d r^{2}}-\frac{k(k+1)}{r^{2}}+\frac{2 k U(r)}{r}-\frac{d U(r)}{r}-U^{2}(r)\right. \\
& -\left[M+E_{n, k}-\Delta(r)\right]\left[M-E_{n, k}+\sum(r)\right] \\
& \left.+\frac{(d \Delta(r) / d r)(d / d r+k / r-U(r))}{\left(M+E_{n, k}-\Delta(r)\right)}\right\} F_{n_{r}, k}(r)=0, \\
& \left\{\frac{d^{2}}{d r^{2}}-\frac{k(k-1)}{r^{2}}+\frac{2 k U(r)}{r}+\frac{d U(r)}{r}-U^{2}(r)\right. \\
& -\left[M+E_{n, k}-\Delta(r)\right]\left[M-E+\sum(r)\right] \\
& \left.+\frac{\left(d \sum(r) / d r\right)(d / d r-k / r+U(r))}{\left(M-E_{n, k}+\sum(r)\right)}\right\} G_{n_{r}, k}(r)=0 .
\end{aligned}
$$

We consider bound state solutions that demand the radial components satisfying $F_{n_{r}, k}(0)=G_{n_{r}, k}(0)=0$, and $F_{n_{r}, k}(\infty)=$ $G_{n_{r}, k}(\infty)=0[45]$.

3.1. Solution Spin Symmetric with Coulomb-Like Tensor Interaction. Under the condition of the spin symmetry-that is, $d \Delta(r) / d r=0$ or $\Delta(r)=C_{s}=$ const-the upper component Dirac equation can be written as

$$
\begin{aligned}
& \left\{\frac{d^{2}}{d r^{2}}-\frac{k(k+1)}{r^{2}}+\frac{2 k U(r)}{r}-\frac{d U(r)}{r}-U^{2}(r)\right. \\
& \left.-\left[M+E_{n, k}-C_{s}\right]\left[M-E_{n, k}+\sum(r)\right]\right\} F_{n_{r}, k}(r) \\
& \quad=0 .
\end{aligned}
$$

The potential $\sum(r)$ is taken as the Eckart $[42,43]$ plus Hulthen potentials [41]:

$$
\begin{aligned}
\sum(r)= & 4 q_{1} \frac{e^{-2 \alpha r}}{\left(1-e^{-2 \alpha r}\right)^{2}}-q_{2} \frac{\left(1+e^{-2 \alpha r}\right)}{\left(1-e^{-2 \alpha r}\right)} \\
& +\frac{v_{0}}{\left(1-e^{-2 \alpha r}\right)}-\frac{v_{1}}{\left(1-e^{-2 \alpha r}\right)^{2}},
\end{aligned}
$$

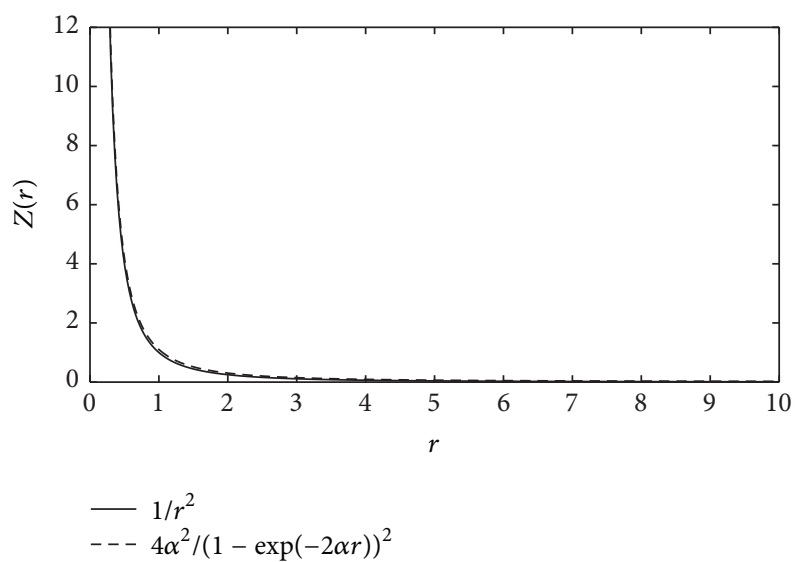

FIGURE 1: The behavior approximation for $\alpha=0.07 \mathrm{fm}^{-1}$.

where the parameters $q_{1}, q_{2}, v_{0}$, and $v_{1}$ are real parameters; these parameters describe the depth of the potential well, and the parameter $\alpha$ is related to the range of the potential.

For the tensor term, we consider the Coulomb-like potential [46],

$$
U(r)=-\frac{H}{r}, \quad H=\frac{Z_{f} Z_{g} e^{2}}{4 \pi \varepsilon_{0}}, r>R_{c}
$$

where $R_{c}$ is the Coulomb radius and $Z_{f}$ and $Z_{g}$ stand for the charges of the projectile particle $f$ and the target nucleus $g$, respectively.

By substituting (11) and (12) into (10), we obtain the upper radial equation of Dirac equation as

$$
\begin{aligned}
& \left\{\frac{d^{2}}{d r^{2}}-\frac{\tau_{k}\left(\tau_{k}+1\right)}{r^{2}}-\gamma-\beta\left(4 q_{1} \frac{e^{-2 \alpha r}}{\left(1-e^{-2 \alpha r}\right)^{2}}\right.\right. \\
& \left.\left.-q_{2} \frac{\left(1+e^{-2 \alpha r}\right)}{\left(1-e^{-2 \alpha r}\right)}+\frac{v_{0}}{\left(1-e^{-2 \alpha r}\right)}-\frac{v_{1}}{\left(1-e^{-2 \alpha r}\right)^{2}}\right)\right\} \\
& \cdot F_{n_{r}, k}(r)=0
\end{aligned}
$$

where $\tau_{k}=k+H, \gamma=\left(M+E_{n, k}-C_{s}\right)\left(M-E_{n, k}\right)$ and $\beta=$ $\left(M+E_{n, k}-C_{s}\right)$.

Equation (13) is exactly solvable only for the case of $k=0$. In order to obtain the analytical solutions of (13), we employ the improved approximation scheme suggested by Greene and Aldrich $[47,48]$ and replace the spin-orbit coupling term with the expression that is valid for $\alpha \leq 1$ [49]:

$$
\frac{1}{r^{2}} \approx \frac{4 \alpha^{2}}{\left(1-e^{-2 \alpha r}\right)^{2}}
$$

The behavior of the improved approximation is plotted in Figure 1 . We can see the good agreement for small $\alpha$ values. 
By applying the transformation $s=\exp (-2 \alpha r)$, (13) is brought into the form

$$
\begin{aligned}
F_{n, k}^{\prime \prime}(s) & +\frac{(1-s)}{s(1-s)} F_{n, k}^{\prime}(s) \\
& +\frac{1}{s^{2}(1-s)^{2}}\left[A s^{2}+B s+C\right] F_{n, k}(s)=0,
\end{aligned}
$$

where the parameters $A, B$, and $C$ are considered as follows:

$$
\begin{aligned}
& A=-\frac{1}{4 \alpha^{2}}\left[\gamma+\beta q_{2}\right] \\
& B=\frac{1}{4 \alpha^{2}}\left[2 \gamma-4 \beta q_{1}+\beta v_{0}\right], \\
& C=-\frac{1}{4 \alpha^{2}}\left[4 \alpha^{2} \tau_{k}\left(\tau_{k}-1\right)+\gamma-\beta q_{2}+\beta v_{0}-\beta v_{1}\right] .
\end{aligned}
$$

$$
\left[\frac{-C-[1 / 2+\sqrt{1 / 4-[A+B+C]}]^{2}-[((1-2 n) / 2)-(1 / 2)(1-\sqrt{-4 A})]^{2}}{2[((1-2 n) / 2)-(1 / 2)(1-\sqrt{-4 A})]}\right]^{2}-\left[\frac{1}{2}+\sqrt{\frac{1}{4}-[A+B+C]}\right]^{2}=0
$$

In Tables 1-3, we give the numerical results for the spin symmetric energy eigenvalues (in units of $\mathrm{fm}^{-1}$ ).

Let us find the corresponding wave functions. In reference to (3) and (18), we can obtain the upper wave function as

$$
\begin{aligned}
& F_{n, k}^{s}(r)=N\left(e^{-2 \alpha r}\right)^{(\sqrt{-c})}\left(1-e^{-2 \alpha r}\right)^{(1 / 2+\sqrt{1 / 4+A+B+C})} \\
& \cdot{ }_{2} F_{1}(-n, n \\
& +2\left(\sqrt{-c}+\frac{1}{2}+\sqrt{\frac{1}{4}+A+B+C}\right) ; 2 \sqrt{-c} \\
& \left.+1, e^{-2 \alpha r}\right)
\end{aligned}
$$

where $N$ is the normalization constant; on the other hand, the lower component of the Dirac spinor can be calculated from

$$
G_{n, k}^{s}(r)=\frac{1}{M+E_{n, k}^{s}-C_{s}}\left(\frac{d}{d r}+\frac{k}{r}-U(r)\right) F_{n, k}^{s}(r)
$$

We have obtained the energy eigenvalues and the wave function of the radial Dirac equation for Eckart plus Hulthen potentials with Coulomb-like tensor interaction in the presence of the spin symmetry for $k \neq 0$.

3.2. Solution Pseudospin Symmetry with Coulomb-Like Tensor Interaction. For pseudospin symmetry-that is,
Now by comparing (15) with (1), we can easily obtain the coefficients $k_{i}(i=1,2,3)$ as follows:

$$
k_{1}=k_{2}=k_{3}=1 \text {. }
$$

The values of the coefficients $k_{i}(i=4,5)$ are also found from (4) as below:

$$
\begin{aligned}
& k_{4}=\sqrt{-C}, \\
& k_{5}=\frac{1}{2}+\sqrt{\frac{1}{4}-[A+B+C]} .
\end{aligned}
$$

Thus, by the use of energy equation (2) for energy eigenvalues, we find $d \sum(r) / d r=0$ or $\sum(r)=C_{\mathrm{ps}}=$ const-the lower component Dirac equation can be written as

$$
\begin{aligned}
& \left\{\frac{d^{2}}{d r^{2}}-\frac{k(k-1)}{r^{2}}+\frac{2 k U(r)}{r}+\frac{d U(r)}{r}-U^{2}(r)\right. \\
& \left.\quad-\left[M+E_{n, k}-\Delta(r)\right]\left[M-E+\sum(r)\right]\right\} G_{n_{r}, k}(r) \\
& \quad=0 .
\end{aligned}
$$

We consider the scalar, vector, and tensor potentials as the following [41]:

$$
\begin{gathered}
\Delta(r)=4 q_{1} \frac{e^{-2 \alpha r}}{\left(1-e^{-2 \alpha r}\right)^{2}}-q_{2} \frac{\left(1+e^{-2 \alpha r}\right)}{\left(1-e^{-2 \alpha r}\right)} \\
+\frac{v_{0}}{\left(1-e^{-2 \alpha r}\right)}-\frac{v_{1}}{\left(1-e^{-2 \alpha r}\right)^{2}}, \\
U(r)=-\frac{H}{r}, \quad H=\frac{Z_{f} Z_{g} e^{2}}{4 \pi \varepsilon_{0}}, r>R_{c} .
\end{gathered}
$$

Substituting (23) into (22), we obtain the lower radial equation of Dirac equation as

$$
\begin{aligned}
& \left\{\frac{d^{2}}{d r^{2}}-\frac{\lambda_{k}\left(\lambda_{k}-1\right)}{r^{2}}-\gamma^{\prime}+\beta^{\prime}\left(4 q_{1} \frac{e^{-2 \alpha r}}{\left(1-e^{-2 \alpha r}\right)^{2}}\right.\right. \\
& \left.\left.-q_{2} \frac{\left(1+e^{-2 \alpha r}\right)}{\left(1-e^{-2 \alpha r}\right)}+\frac{v_{0}}{\left(1-e^{-2 \alpha r}\right)}-\frac{v_{1}}{\left(1-e^{-2 \alpha r}\right)^{2}}\right)\right\} \\
& \cdot G_{n_{r}, k}(r)=0,
\end{aligned}
$$


TABLE 1: Energies of the spin symmetry limit in the presence and absence of Coulomb-like tensor interaction by parameters $M=10 \mathrm{fm}^{-1}$, $c=1, h=1, \alpha=0.05 \mathrm{fm}^{-1}, V_{1}=0.4 \mathrm{fm}^{-1}, V_{0}=0.3 \mathrm{fm}^{-1}, q_{1}=0.1 \mathrm{fm}^{-1}, q_{2}=0.2 \mathrm{fm}^{-1}$, and $C_{s}=5 \mathrm{fm}^{-1}$.

\begin{tabular}{ccccccccc}
\hline$l$ & $n, k>0$ & State $(l, j)$ & $E_{n, k}^{s}(H=0)$ & $E_{n, k}^{s}(H=0.65)$ & $n, k<0$ & State $(l, j+1)$ & $E_{n, k}^{s}(H=0)$ & $E_{n, k}^{s}(H=0.65)$ \\
\hline 1 & 1,1 & $1 \mathrm{p}_{1 / 2}$ & 3.854830541 & 5.230932502 & $1,-2$ & $1 \mathrm{p}_{3 / 2}$ & 3.854830541 & 2.022333483 \\
2 & 1,2 & $1 \mathrm{~d}_{3 / 2}$ & 5.816938791 & 6.683341758 & $1,-3$ & $1 \mathrm{~d}_{5 / 2}$ & 5.816938791 & 4.646742373 \\
3 & 1,3 & $1 \mathrm{f}_{5 / 2}$ & 7.054878462 & 7.612226007 & $1,-4$ & $1 \mathrm{f}_{7 / 2}$ & 7.054878462 & 6.315225843 \\
4 & 1,4 & $1 \mathrm{~g}_{7 / 2}$ & 7.855626422 & 8.227670602 & $1,-5$ & $1 \mathrm{~g}_{9 / 2}$ & 7.855626422 & 7.373922639 \\
1 & 2,1 & $2 \mathrm{p}_{1 / 2}$ & 5.770989098 & 6.634606185 & $2,-2$ & $2 \mathrm{p}_{3 / 2}$ & 5.770989098 & 4.607350329 \\
2 & 2,2 & $2 \mathrm{~d}_{3 / 2}$ & 7.005594455 & 7.562996503 & $2,-3$ & $2 \mathrm{~d}_{5 / 2}$ & 7.005594455 & 6.267433123 \\
3 & 2,3 & $2 \mathrm{f}_{5 / 2}$ & 7.806806149 & 8.180021794 & $2,-4$ & $2 \mathrm{f}_{7 / 2}$ & 7.806806149 & 7.324529686 \\
4 & 2,4 & $2 \mathrm{~g}_{7 / 2}$ & 8.346470004 & 8.605903663 & $2,-5$ & $2 \mathrm{~g}_{9 / 2}$ & 8.346470004 & 8.019210395 \\
\hline
\end{tabular}

TABLE 2: The energy eigenvalues (in units of $\mathrm{fm}^{-1}$ ) for the spin symmetry limit with parameters $M=10 \mathrm{fm}^{-1}, c=1, h=1, V_{1}$ $=4 \mathrm{fm}^{-1}, V_{0}=3 \mathrm{fm}^{-1}, q_{1}=1 \mathrm{fm}^{-1}, q_{2}=2 \mathrm{fm}^{-1}$, and $C_{s}=5 \mathrm{fm}^{-1}$.

\begin{tabular}{lcccc}
\hline \multirow{2}{*}{$\alpha\left(\mathrm{fm}^{-1}\right)$} & \multicolumn{2}{c}{$E_{n, k}(H=0)$} & \multicolumn{2}{c}{$E_{n, k}(H=0.65)$} \\
& $1 \mathrm{~d}_{3 / 2}$ & $1 \mathrm{~d}_{5 / 2}$ & $1 \mathrm{~d}_{3 / 2}$ & $1 \mathrm{~d}_{5 / 2}$ \\
\hline 0.3 & 2.367211785 & 2.367211785 & 3.624581074 & 0.93609384 \\
0.35 & 3.53989514 & 3.53989514 & 4.771398349 & 2.070547382 \\
0.4 & 4.521194951 & 4.521194951 & 5.690996586 & 3.069929104 \\
0.5 & 5.998968975 & 5.998968975 & 7.009500454 & 4.671333928 \\
0.6 & 6.995852039 & 6.995852039 & 7.853318047 & 5.827299953 \\
0.7 & 7.670572507 & 7.670572507 & 8.400594789 & 6.652859598 \\
\hline
\end{tabular}

TABLE 3: The energy eigenvalues (in units of $\mathrm{fm}^{-1}$ ) for the spin symmetry limit with parameters $c=1, h=1, \alpha=0.05 \mathrm{fm}^{-1}, V_{1}$ $=4 \mathrm{fm}^{-1}, V_{0}=3 \mathrm{fm}^{-1}, q_{1}=1 \mathrm{fm}^{-1}, q_{2}=2 \mathrm{fm}^{-1}$, and $C_{s}=5 \mathrm{fm}^{-1}$.

\begin{tabular}{lcccc}
\hline \multirow{2}{*}{$M\left(\mathrm{fm}^{-1}\right)$} & \multicolumn{2}{c}{$E_{n, k}(H=0)$} & \multicolumn{2}{c}{$E_{n, k}(H=0.65)$} \\
& $1_{3 / 2}$ & $1_{5 / 2}$ & $1 \mathrm{~d}_{3 / 2}$ & $1 \mathrm{~d}_{5 / 2}$ \\
\hline 5.5 & 3.335677895 & 3.335677895 & 3.893525562 & 2.662575645 \\
6 & 3.479942785 & 3.479942785 & 4.105505756 & 2.72071499 \\
6.5 & 3.618211096 & 3.618211096 & 4.311706034 & 2.7725715 \\
7 & 3.752572889 & 3.752572889 & 4.514086551 & 2.820419493 \\
8 & 4.013951033 & 4.013951033 & 4.911575769 & 2.908709541 \\
9 & 4.269356779 & 4.269356779 & 5.303088298 & 2.991067928 \\
10 & 4.521194951 & 4.521194951 & 5.690996586 & 3.069929104 \\
\hline
\end{tabular}

where $\lambda_{k}=k+H, \gamma^{\prime}=\left(M+E_{n, k}\right)\left(M-E_{n, k}+C_{\mathrm{ps}}\right)$ and $\beta^{\prime}=\left(M-E_{n, k}+C_{\mathrm{ps}}\right)$.
By using the transformation $s=\exp (-2 \alpha r)$ and employing the improved approximation, (24) is brought into the form

$$
\begin{aligned}
G_{n, k}^{\prime \prime}(s) & +\frac{(1-s)}{s(1-s)} G_{n, k}^{\prime}(s) \\
& +\frac{1}{s^{2}(1-s)^{2}}\left[A^{\prime} s^{2}+B^{\prime} s+C^{\prime}\right] G_{n, k}(s)=0
\end{aligned}
$$

where the parameters $A^{\prime}, B^{\prime}$, and $C^{\prime}$ are considered as follows:

$$
\begin{aligned}
& A^{\prime}=-\frac{1}{4 \alpha^{2}}\left[\gamma^{\prime}-\beta^{\prime} q_{2}\right], \\
& B^{\prime}=\frac{1}{4 \alpha^{2}}\left[2 \gamma^{\prime}+4 \beta^{\prime} q_{1}-\beta^{\prime} v_{0}\right], \\
& C^{\prime} \\
& =-\frac{1}{4 \alpha^{2}}\left[4 \alpha^{2} \lambda_{k}\left(\lambda_{k}-1\right)+\gamma^{\prime}+\beta^{\prime} q_{2}-\beta^{\prime} v_{0}+\beta^{\prime} v_{1}\right] .
\end{aligned}
$$

We can easily obtain the coefficients $k_{i}(i=1,2,3)$ by comparing (25) with (1) as follows:

$$
k_{1}^{\prime}=k_{2}^{\prime}=k_{3}^{\prime}=1 \text {. }
$$

The values of the coefficients $k_{i}^{\prime}(i=4,5)$ are also found from (4) as below:

$$
\begin{aligned}
& k_{4}^{\prime}=\sqrt{-C^{\prime}}, \\
& k_{5}^{\prime}=\frac{1}{2}+\sqrt{\frac{1}{4}-\left[A^{\prime}+B^{\prime}+C^{\prime}\right]} .
\end{aligned}
$$

We have (29) for energy eigenvalues by the use of (2):

$$
\begin{aligned}
& {\left[\frac{-C^{\prime}-\left[1 / 2+\sqrt{1 / 4-\left[A^{\prime}+B^{\prime}+C^{\prime}\right]}\right]^{2}-\left[((1-2 n) / 2)-(1 / 2)\left(1-\sqrt{-4 A^{\prime}}\right)\right]^{2}}{2\left[((1-2 n) / 2)-(1 / 2)\left(1-\sqrt{-4 A^{\prime}}\right)\right]}\right]^{2}} \\
& -\left[\frac{1}{2}+\sqrt{\frac{1}{4}-\left[A^{\prime}+B^{\prime}+C^{\prime}\right]}\right]^{2}=0 .
\end{aligned}
$$


TABLE 4: The energy eigenvalues (in units of $\mathrm{fm}^{-1}$ ) for the pseudospin symmetry limit in the presence and absence of Coulomb-like tensor interaction: $M=10 \mathrm{fm}^{-1}, c=1, \hbar=1, \alpha=0.05 \mathrm{fm}^{-1}, V_{1}=0.4 \mathrm{fm}^{-1}, V_{0}=0.3 \mathrm{fm}^{-1}, q_{1}=0.1 \mathrm{fm}^{-1}, q_{2}=-0.2 \mathrm{fm}^{-1}$, and $C_{\mathrm{ps}}=-5 \mathrm{fm}^{-1}$.

\begin{tabular}{ccccccccc}
\hline$l$ & $n, k<0$ & State $(l, j)$ & $E_{n, k}^{\mathrm{ps}}(H=0)$ & $E_{n, k}^{\mathrm{ps}}(H=0.65)$ & $n, k>0$ & State $(l+2, j+1)$ & $E_{n, k}^{\mathrm{ps}}(H=0)$ & $E_{n, k}^{\mathrm{ps}}(H=0.65)$ \\
\hline 1 & $1,-1$ & $1 \mathrm{~s}_{1 / 2}$ & -7.065764205 & -5.701645852 & 1,2 & $1 \mathrm{~d}_{3 / 2}$ & -7.065764205 & -7.916615587 \\
2 & $1,-2$ & $1 \mathrm{p}_{3 / 2}$ & -8.242801196 & -7.570912051 & 1,3 & $1 \mathrm{f}_{5 / 2}$ & -8.242801196 & -8.691210574 \\
3 & $1,-3$ & $1 \mathrm{~d}_{5 / 2}$ & -8.872276828 & -8.505344072 & 1,4 & $1 \mathrm{~g}_{7 / 2}$ & -8.872276828 & -9.132415328 \\
4 & $1,-4$ & $1 \mathrm{f}_{7 / 2}$ & -9.241962415 & -9.022813407 & 1,5 & $1 \mathrm{~h}_{9 / 2}$ & -9.241962415 & -9.404940153 \\
1 & $2,-1$ & $2 \mathrm{~s}_{1 / 2}$ & -8.20892624 & -7.540097339 & 2,2 & $2 \mathrm{~d}_{3 / 2}$ & -8.20892624 & -8.656719162 \\
2 & $2,-2$ & $2 \mathrm{p}_{3 / 2}$ & -8.837973611 & -8.470939351 & 2,3 & $2 \mathrm{f}_{5 / 2}$ & -8.837973611 & -9.098960172 \\
3 & $2,-3$ & $2 \mathrm{~d}_{5 / 2}$ & -9.209115205 & -8.988907697 & 2,4 & $2 \mathrm{~g}_{7 / 2}$ & -9.209115205 & -9.37334647 \\
4 & $2,-4$ & $2 \mathrm{f}_{7 / 2}$ & -9.44496483 & -9.303214658 & 2,5 & $2 \mathrm{~h}_{9 / 2}$ & -9.44496483 & -9.554705712 \\
\hline
\end{tabular}

In Tables 4-6, we give the numerical results for the pseudospin symmetric energy eigenvalues (in units of $\mathrm{fm}^{-1}$ ).

By using (3) and (28), we can finally obtain the lower component of the Dirac spinor as below:

$$
\begin{aligned}
& G_{n, k}^{\mathrm{ps}}(r)=N\left(e^{-2 \alpha r}\right)^{\left(\sqrt{-c^{\prime}}\right)}(1 \\
& \left.-e^{-2 \alpha r}\right)^{\left(1 / 2+\sqrt{1 / 4+A^{\prime}+B^{\prime}+C^{\prime}}\right)}{ }_{2} F_{1}(-n, n \\
& +2\left(\sqrt{-c^{\prime}}+\frac{1}{2}+\sqrt{\frac{1}{4}+A^{\prime}+B^{\prime}+C^{\prime}}\right) ; 2 \sqrt{-c^{\prime}} \\
& \left.+1, e^{-2 \alpha r}\right)
\end{aligned}
$$

where $N^{\prime}$ is the normalization constant; on the other hand, the upper component of the Dirac spinor can be calculated from (30) as

$$
F_{n, k}^{\mathrm{ps}}(r)=\frac{1}{M-E_{n, k}^{\mathrm{ps}}+C_{\mathrm{ps}}}\left(\frac{d}{d r}-\frac{k}{r}+U(r)\right) G_{n, k}^{\mathrm{ps}}(r) .
$$

We have obtained the energy eigenvalues and the spinors of the radial Dirac equation for Eckart plus Hulthen potentials with Coulomb-like tensor interaction in the presence of the pseudospin symmetry for $k \neq 0$. We show in Figure 2 behavior energy for various $H$ in the spin and pseudospin symmetry.

3.3. Solution Spin Symmetry with Coulomb Plus Yukawa-Like Tensor Interaction. In this section, for the spin symmetry, we consider $\Delta(r)=C_{s}, \sum(r)$, and $U(r)$ as the following:

$$
\begin{aligned}
\sum(r)= & 4 q_{1} \frac{e^{-2 \alpha r}}{\left(1-e^{-2 \alpha r}\right)^{2}}-q_{2} \frac{\left(1+e^{-2 \alpha r}\right)}{\left(1-e^{-2 \alpha r}\right)} \\
& +\frac{v_{0}}{\left(1-e^{-2 \alpha r}\right)}-\frac{v_{1}}{\left(1-e^{-2 \alpha r}\right)^{2}}, \\
U(r)= & -\frac{H}{r}-A \frac{\exp (-2 \alpha r)}{r},
\end{aligned}
$$

TABLE 5: The energy eigenvalues (in units of $\mathrm{fm}^{-1}$ ) for the pseudospin symmetry limit with parameters $M=10 \mathrm{fm}^{-1}, c=1, \hbar=1$, $V_{1}=4 \mathrm{fm}^{-1}, V_{0}=3 \mathrm{fm}^{-1}, q_{1}=1 \mathrm{fm}^{-1}, q_{2}=-2 \mathrm{fm}^{-1}$, and $C_{\mathrm{ps}}=-5 \mathrm{fm}^{-1}$.

\begin{tabular}{lcccc}
\hline$\alpha$ & \multicolumn{2}{c}{$E_{n, k}^{\mathrm{ps}}(H=0)$} & \multicolumn{2}{c}{$E_{n, k}^{\mathrm{ps}}(H=0.65)$} \\
$\left(\mathrm{fm}^{-1}\right)$ & $1 \mathrm{~s}_{1 / 2}$ & $1 \mathrm{~d}_{3 / 2}$ & $1 \mathrm{~s}_{1 / 2}$ & $1 \mathrm{~d}_{3 / 2}$ \\
\hline 0.3 & -4.313211747 & -4.313211747 & -2.304589779 & -5.851610879 \\
0.35 & -5.441940815 & -5.441940815 & -3.496832686 & -6.846892358 \\
0.4 & -6.330481794 & -6.330481794 & -4.501756572 & -7.592836004 \\
0.5 & -7.58011028 & -7.58011028 & -6.029844453 & -8.587003959 \\
0.6 & -8.366177606 & -8.366177606 & -7.07440217 & -9.176918108 \\
0.7 & -8.871669319 & -8.871669319 & -7.792319679 & -9.537028876 \\
\hline
\end{tabular}

where $H$ and $A$ are the real parameters; substitution of (32) into (10) yields

$$
\begin{aligned}
& F_{n, k}^{\prime \prime}(s)+\frac{(1-s)}{s(1-s)} F_{n, k}^{\prime}(s) \\
& \quad+\frac{1}{s^{2}(1-s)^{2}}\left[\chi_{2} s^{2}+\chi_{1} s+\chi_{0}\right] F_{n, k}(s)=0
\end{aligned}
$$

where the parameters $\chi_{2}, \chi_{1}$, and $\chi_{0}$ are considered as follows:

$$
\begin{aligned}
& \chi_{2}=-\frac{1}{4 \alpha^{2}}\left[\gamma+\beta q_{2}\right]+A(A-1), \\
& \chi_{1}=\frac{1}{4 \alpha^{2}}\left[2 \gamma-4 \beta q_{1}+\beta v_{0}\right]-2 A \tau_{k}, \\
& \chi_{0}=-\frac{1}{4 \alpha^{2}}\left[4 \alpha^{2} \tau_{k}\left(\tau_{k}-1\right)+\gamma-\beta q_{2}+\beta v_{0}-\beta v_{1}\right] .
\end{aligned}
$$

By comparing (33) with (1), we can easily obtain the coefficients $k_{i}(i=1,2,3)$ as follows:

$$
k_{1}=k_{2}=k_{3}=1 \text {. }
$$

The values of the coefficients $k_{i}(i=4,5)$ are also found from (4) as below:

$$
\begin{aligned}
& k_{4}=\sqrt{-\chi_{0}} \\
& k_{5}=\frac{1}{2}+\sqrt{\frac{1}{4}-\left[\chi_{2}+\chi_{1}+\chi_{0}\right]} .
\end{aligned}
$$




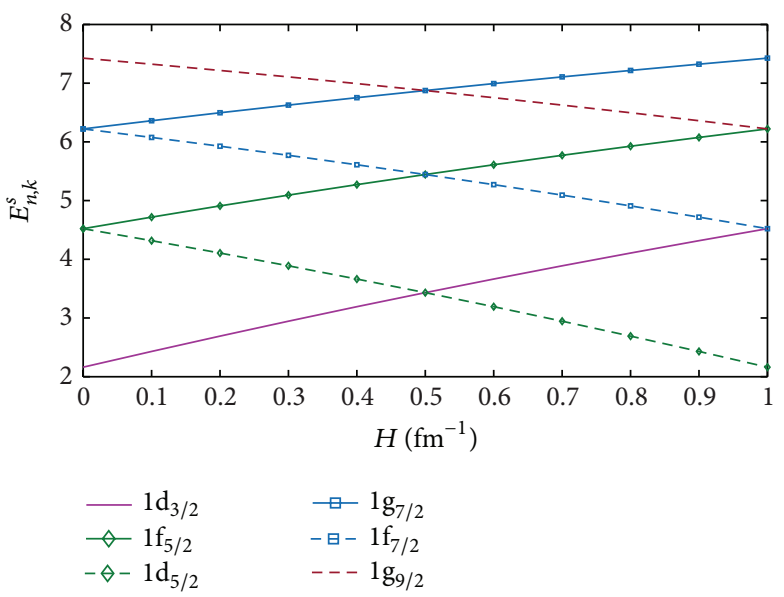

(a)

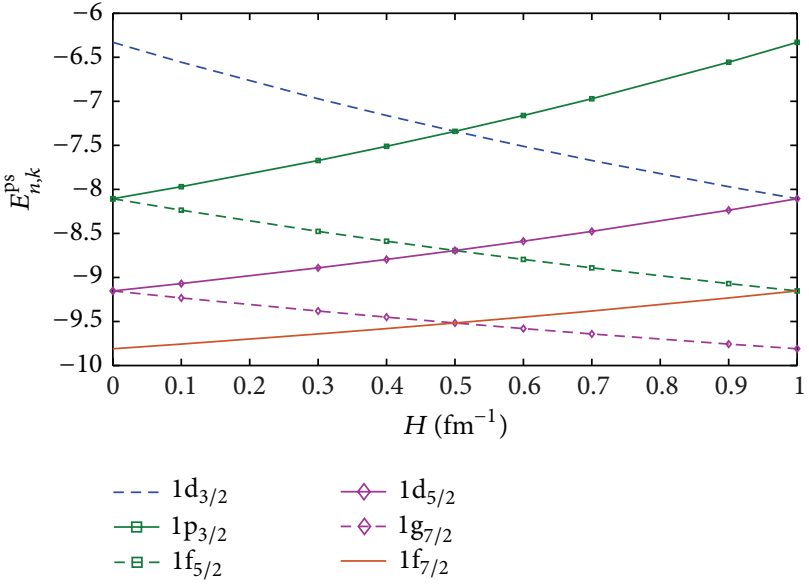

(b)

FIGURE 2: Energy spectra in the (a) spin and (b) pseudospin symmetries at various $H$ for Coulomb-tensor interaction with parameters $M=10 \mathrm{fm}^{-1}, c=1, \hbar=1, \alpha=0.4 \mathrm{fm}^{-1}, V_{1}=4 \mathrm{fm}^{-1}, V_{0}=3 \mathrm{fm}^{-1}, q_{1}=1 \mathrm{fm}^{-1}, q_{2}^{\mathrm{ps}}=-2 \mathrm{fm}^{-1}, q_{2}^{s}=2 \mathrm{fm}^{-1}, C_{s}=5 \mathrm{fm}^{-1}$, and $C_{\mathrm{ps}}=-5 \mathrm{fm}^{-1}$.

TABLE 6: The energy eigenvalues (in units of $\mathrm{fm}^{-1}$ ) for the pseudospin symmetry limit with parameters $c=1, \hbar=1, \alpha=0.4 \mathrm{fm}^{-1}, V_{1}=4 \mathrm{fm}^{-1}$, $V_{0}=3 \mathrm{fm}^{-1}, q_{1}=1 \mathrm{fm}^{-1}, q_{2}=-2 \mathrm{fm}^{-1}$, and $C_{\mathrm{ps}}=-5 \mathrm{fm}^{-1}$.

\begin{tabular}{lcccc}
\hline$M\left(\mathrm{fm}^{-1}\right)$ & \multicolumn{1}{c}{$E_{n, k}^{\mathrm{ps}}(H=0)$} & \multicolumn{2}{c}{$E_{n, k}^{\mathrm{ps}}(H=0.65)$} & $1_{1 / 2}$ \\
\hline 5.5 & $1 \mathrm{~s}_{1 / 2}$ & -4.282887209 & -3.435345049 & -4.891201159 \\
6 & -4.282887209 & -4.519466496 & -3.562129097 & -5.200846041 \\
6.5 & -4.519466496 & -4.751863076 & -3.685040453 & -5.506165891 \\
7 & -4.751863076 & -4.981434942 & -3.80536706 & -5.808539775 \\
8 & -4.981434942 & -5.435100161 & -4.04106051 & -6.407528709 \\
9 & -5.435100161 & -5.884189891 & -4.27265172 & -7.001673282 \\
10 & -5.884189891 & -6.330481794 & -4.501756572 & -7.592836004 \\
\hline
\end{tabular}

By the use of energy equation (2) for energy eigenvalues, we have

$$
\left[\frac{\left.-\chi_{0}-\left[1 / 2+\sqrt{1 / 4-\left[\chi_{2}+\chi_{1}+\chi_{0}\right.}\right]\right]^{2}-\left[((1-2 n) / 2)-(1 / 2)\left(1-\sqrt{-4 \chi_{2}}\right)\right]^{2}}{2\left[((1-2 n) / 2)-(1 / 2)\left(1-\sqrt{-4 \chi_{2}}\right)\right]}\right]^{2}-\left[\frac{1}{2}+\sqrt{\frac{1}{4}-\left[\chi_{2}+\chi_{1}+\chi_{0}\right]}\right]^{2}=0
$$

In reference to (3) and using (36), we can obtain the upper wave function:

$$
\begin{aligned}
& F_{n, k}^{s}(r)=N\left(e^{-2 \alpha r}\right)^{\left(\sqrt{-\chi_{0}}\right)}(1 \\
& \left.-e^{-2 \alpha r}\right)^{\left(1 / 2+\sqrt{1 / 4+\chi_{2}+\chi_{1}+\chi_{0}}\right)}{ }_{2} F_{1}(-n, n \\
& \quad+2\left(\sqrt{-\chi_{0}}+\frac{1}{2}+\sqrt{\frac{1}{4}+\chi_{2}+\chi_{1}+\chi_{0}}\right) ; 2 \sqrt{-\chi_{0}}
\end{aligned}
$$

$$
\left.+1, e^{-2 \alpha r}\right)
$$

where $N$ is the normalization constant; on the other hand, the upper component of the Dirac spinor can be calculated from (38) as

$$
G_{n, k}^{s}(r)=\frac{1}{M+E_{n, k}^{s}-C_{s}}\left(\frac{d}{d r}+\frac{k}{r}-U(r)\right) F_{n, k}^{s}(r) .
$$




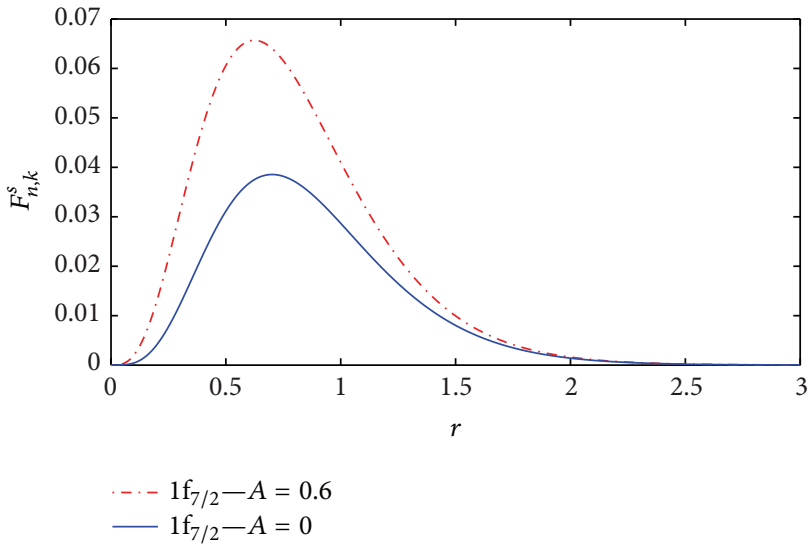

(a)

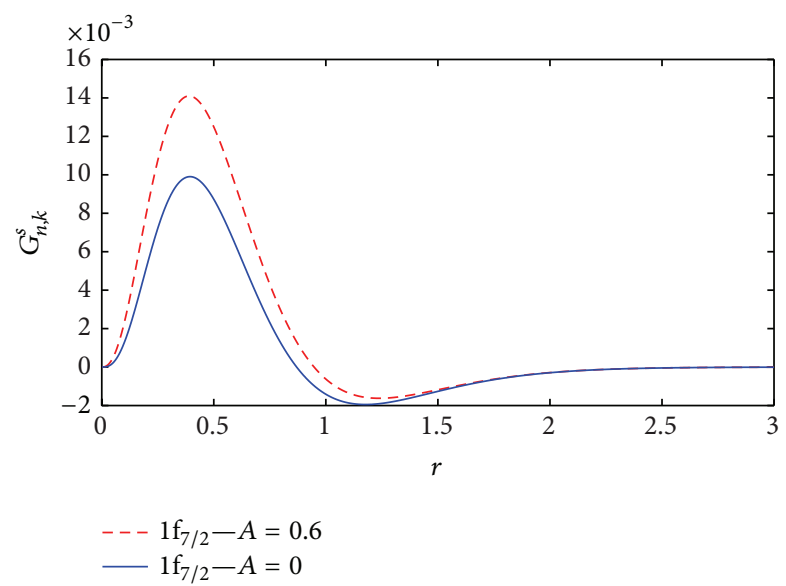

(b)

FIGURE 3: (a) Upper and (b) lower components of $1 \mathrm{f}_{7 / 2}$ in the spin symmetry in the presence and absence of Yukawa tensor interaction for $M=10 \mathrm{fm}^{-1}, c=1, \hbar=1, \alpha=0.4 \mathrm{fm}^{-1}, V_{1}=4 \mathrm{fm}^{-1}, V_{0}=3 \mathrm{fm}^{-1}, q_{1}=1 \mathrm{fm}^{-1}, q_{2}=2 \mathrm{fm}^{-1}$, and $C_{s}=5 \mathrm{fm}^{-1}$.

The effects of the Yukawa-like tensor interactions on the upper and lower components for the spin symmetry are shown in Figure 3.

We have obtained the energy eigenvalues and the spinors of the radial Dirac equation for Eckart plus Hulthen potentials with the spin symmetry for $k \neq 0$ in the presence and absence of Yukawa tensor.

3.4. Solution Pseudospin Symmetry with Coulomb Plus Yukawa-Like Tensor Interaction. In this section, for the pseudospin symmetry, we consider $\sum(r)=C_{s}, \Delta(r)$, and $U(r)$ as the following:

$$
\begin{aligned}
\Delta(r)= & 4 q_{1} \frac{e^{-2 \alpha r}}{\left(1-e^{-2 \alpha r}\right)^{2}}-q_{2} \frac{\left(1+e^{-2 \alpha r}\right)}{\left(1-e^{-2 \alpha r}\right)} \\
& +\frac{v_{0}}{\left(1-e^{-2 \alpha r}\right)}-\frac{v_{1}}{\left(1-e^{-2 \alpha r}\right)^{2}}, \\
U(r)= & -\frac{H}{r}-A \frac{\exp (-2 \alpha r)}{r},
\end{aligned}
$$

where $H$ and $A$ are the real parameters. Substitution of (40) into (22) yields

$$
\begin{aligned}
G_{n, k}^{\prime \prime}(s) & +\frac{(1-s)}{s(1-s)} G_{n, k}^{\prime}(s) \\
& +\frac{1}{s^{2}(1-s)^{2}}\left[\chi_{2}^{\prime} s^{2}+\chi_{1}^{\prime} s+\chi_{0}^{\prime}\right] G_{n, k}(s)=0,
\end{aligned}
$$

where the parameters $\chi_{2}^{\prime}, \chi_{1}^{\prime}$, and $\chi_{0}^{\prime}$ are considered as follows:

$$
\begin{aligned}
& \chi_{2}^{\prime}=-\frac{1}{4 \alpha^{2}}\left[\gamma-\beta q_{2}\right]+A(A+1), \\
& \chi_{1}^{\prime}=\frac{1}{4 \alpha^{2}}\left[2 \gamma+4 \beta q_{1}-\beta v_{0}\right]-2 A\left(\lambda_{k}-1\right), \\
& \chi_{0}^{\prime}=-\frac{1}{4 \alpha^{2}}\left[4 \alpha^{2} \lambda_{k}\left(\lambda_{k}-1\right)+\gamma+\beta q_{2}-\beta v_{0}+\beta v_{1}\right] .
\end{aligned}
$$

By comparing (41) with (1), we can easily obtain the coefficients $k_{i}^{\prime}(i=1,2,3)$ as follows:

$$
k_{1}^{\prime}=k_{2}^{\prime}=k_{3}^{\prime}=1 .
$$

The values of the coefficients $k_{i}^{\prime}(i=4,5)$ are also found from (4) as below:

$$
\begin{aligned}
& k_{4}^{\prime}=\sqrt{-\chi_{0}^{\prime}}, \\
& k_{5}^{\prime}=\frac{1}{2}+\sqrt{\frac{1}{4}-\left[\chi_{2}^{\prime}+\chi_{1}^{\prime}+\chi_{0}^{\prime}\right]} .
\end{aligned}
$$

By using energy equation (2) for energy eigenvalues, we have

$$
\begin{aligned}
& {\left[\frac{\left.-\chi_{0}^{\prime}-\left[1 / 2+\sqrt{1 / 4-\left[\chi_{2}^{\prime}+\chi_{1}^{\prime}+\chi_{0}^{\prime}\right.}\right]\right]^{2}-\left[((1-2 n) / 2)-(1 / 2)\left(1-\sqrt{-4 \chi_{2}^{\prime}}\right)\right]^{2}}{2\left[((1-2 n) / 2)-(1 / 2)\left(1-\sqrt{-4 \chi_{2}^{\prime}}\right)\right]}\right]^{2}} \\
& -\left[\frac{1}{2}+\sqrt{\frac{1}{4}-\left[\chi_{2}^{\prime}+\chi_{1}^{\prime}+\chi_{0}^{\prime}\right]}\right]^{2}=0 .
\end{aligned}
$$




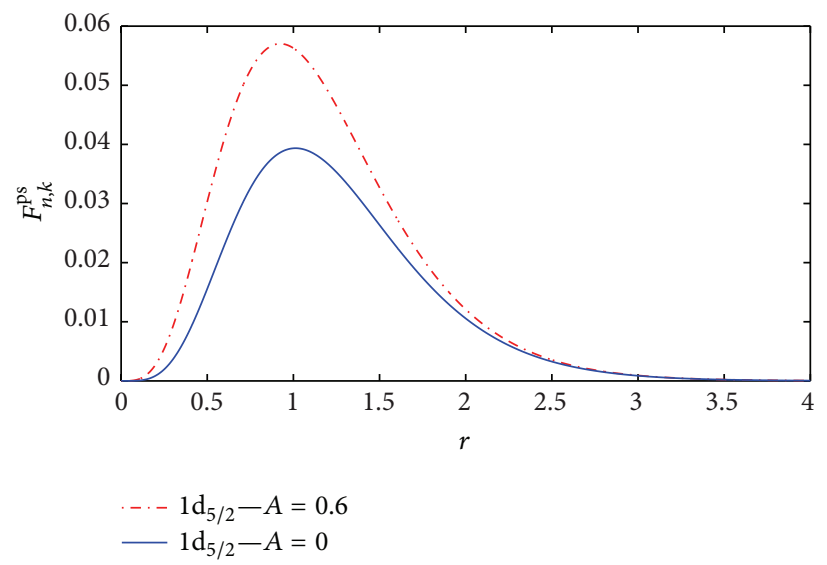

(a)

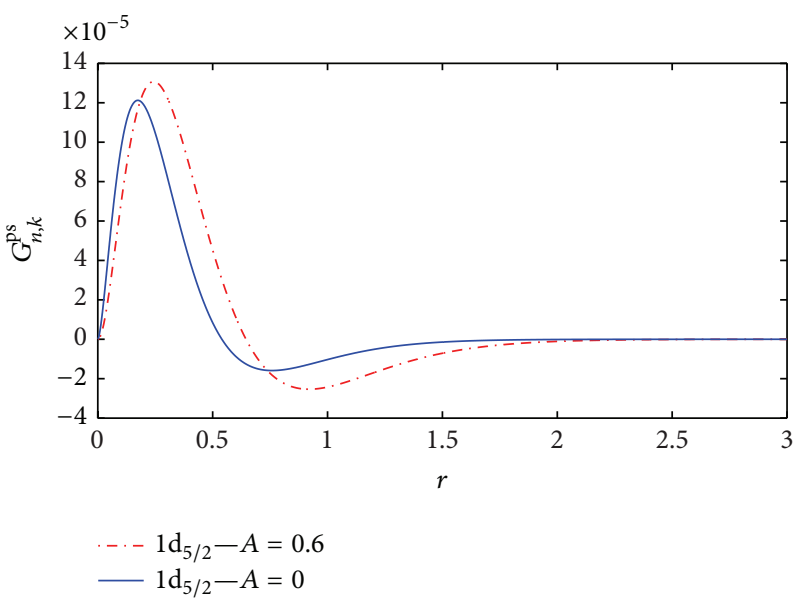

(b)

FIGURE 4: (a) Upper and (b) lower components of $1 \mathrm{~d}_{5 / 2}$ in the pseudospin symmetry in the presence and absence of Yukawa tensor interaction for $M=10 \mathrm{fm}^{-1}, c=1, \hbar=1, \alpha=0.4 \mathrm{fm}^{-1}, V_{1}=4 \mathrm{fm}^{-1}, V_{0}=3 \mathrm{fm}^{-1}, q_{1}=1 \mathrm{fm}^{-1}, q_{2}=-2 \mathrm{fm}^{-1}$, and $C_{\mathrm{ps}}=-5 \mathrm{fm}^{-1}$.

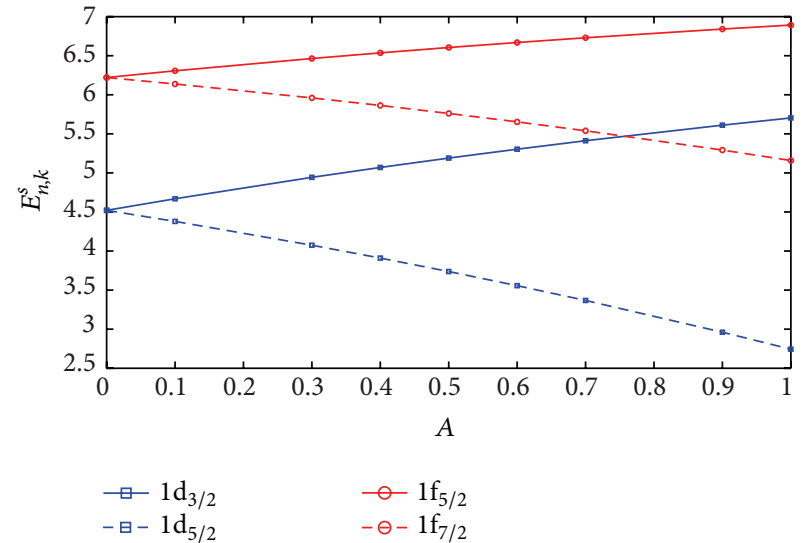

(a)

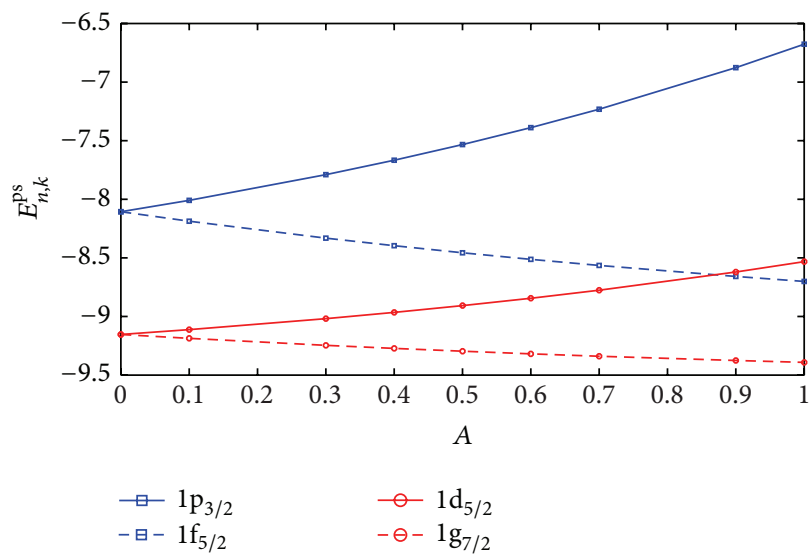

(b)

Figure 5: Energy spectra in the (a) spin and (b) pseudospin symmetries versus $A$ for Yukawa-like tensor interaction with parameters $M=$ $10 \mathrm{fm}^{-1}, c=1, \hbar=1, \alpha=0.4 \mathrm{fm}^{-1}, V_{1}=4 \mathrm{fm}^{-1}, V_{0}=3 \mathrm{fm}^{-1}, q_{1}=1 \mathrm{fm}^{-1}, q_{2}^{\mathrm{ps}}=-2 \mathrm{fm}^{-1}, q_{2}^{s}=2 \mathrm{fm}^{-1}, C_{s}=5 \mathrm{fm}^{-1}$, and $C_{\mathrm{ps}}=-5 \mathrm{fm}^{-1}$.

By the use of (3) and (44), we can finally obtain the lower component of the Dirac spinor as below:

$$
\begin{aligned}
& G_{n, k}^{\mathrm{ps}}(r)=N\left(e^{-2 \alpha r}\right)^{\left(\sqrt{-\chi_{0}^{\prime}}\right)}(1 \\
& \left.\quad-e^{-2 \alpha r}\right)^{\left(1 / 2+\sqrt{1 / 4+\chi_{2}^{\prime}+\chi_{1}^{\prime}+\chi_{0}^{\prime}}\right)}{ }_{2} F_{1}(-n, n \\
& \quad+2\left(\sqrt{-\chi_{0}^{\prime}}+\frac{1}{2}+\sqrt{\frac{1}{4}+\chi_{2}^{\prime}+\chi_{1}^{\prime}+\chi_{0}^{\prime}}\right) ; 2 \sqrt{-\chi_{0}^{\prime}} \\
& \left.+1, e^{-2 \alpha r}\right),
\end{aligned}
$$

where $N^{\prime}$ is the normalization constant; on the other hand, the upper component of the Dirac spinor can be calculated from (46) as

$$
\begin{aligned}
F_{n, k}^{\mathrm{ps}} & (r) \\
\quad & =\frac{1}{M-E_{n, k}^{\mathrm{ps}}+C_{\mathrm{ps}}}\left(\frac{d}{d r}-\frac{k}{r}+U(r)\right) G_{n, k}^{\mathrm{ps}}(r) .
\end{aligned}
$$

The effects of the Yukawa-like tensor interactions on the upper and lower components for the pseudospin symmetry are shown in Figure 4.

We have obtained the energy eigenvalues and the spinors of the radial Dirac equation for Eckart plus Hulthen potentials with the pseudospin symmetry for $k \neq 0$ in the presence and absence of Yukawa tensor interaction. In Figures 5 and 6, 


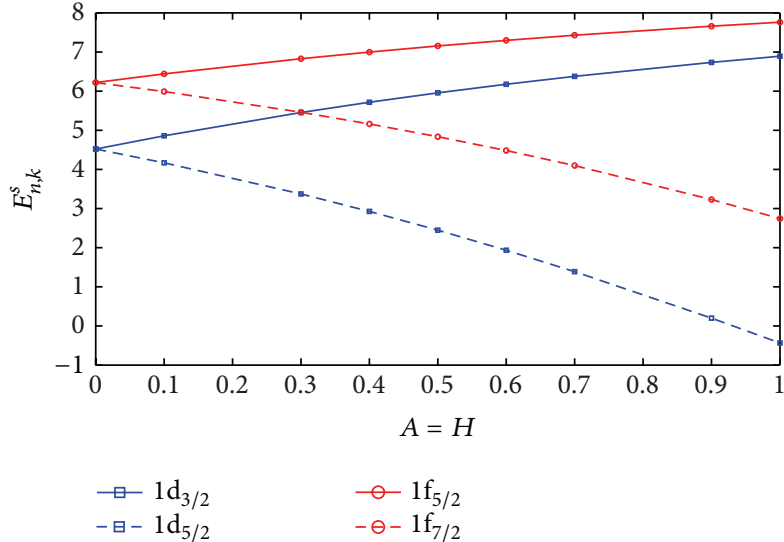

(a)

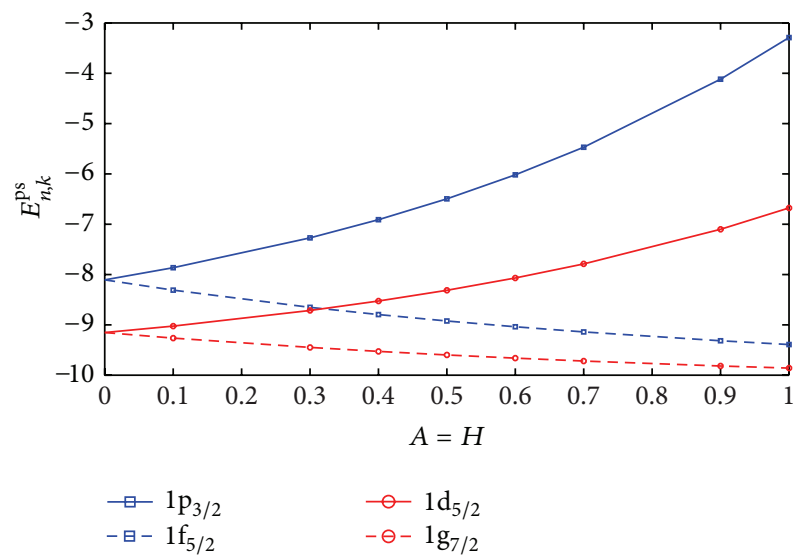

(b)

FiguRE 6: Energy spectra in the (a) spin and (b) pseudospin symmetries versus $A=H$ for Yukawa plus Coulomb-like tensor interaction with parameters $M=10 \mathrm{fm}^{-1}, c=1, \hbar=1, \alpha=0.4 \mathrm{fm}^{-1}, V_{1}=4 \mathrm{fm}^{-1}, V_{0}=3 \mathrm{fm}^{-1}, q_{1}=1 \mathrm{fm}^{-1}, q_{2}^{\mathrm{ps}}=-2 \mathrm{fm}^{-1}, q_{2}^{s}=2 \mathrm{fm}^{-1}, C_{s}=5 \mathrm{fm}^{-1}$, and $C_{\mathrm{ps}}=-5 \mathrm{fm}^{-1}$.

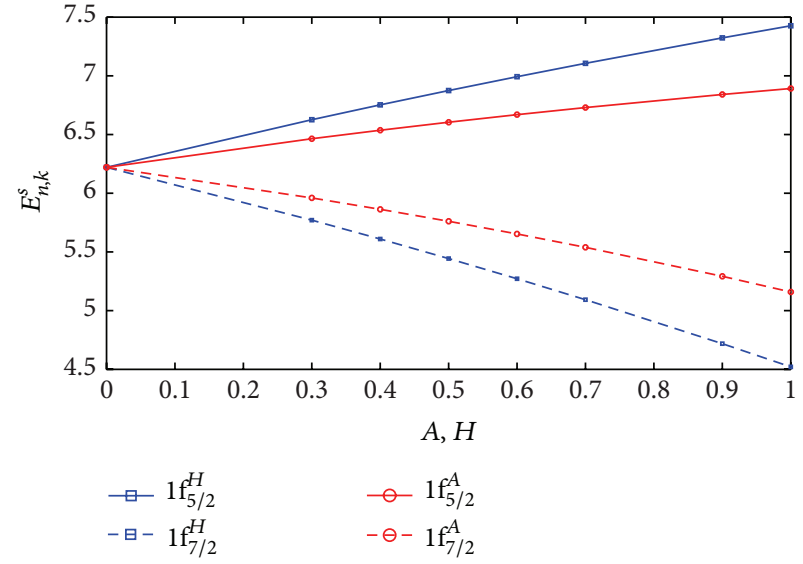

(a)

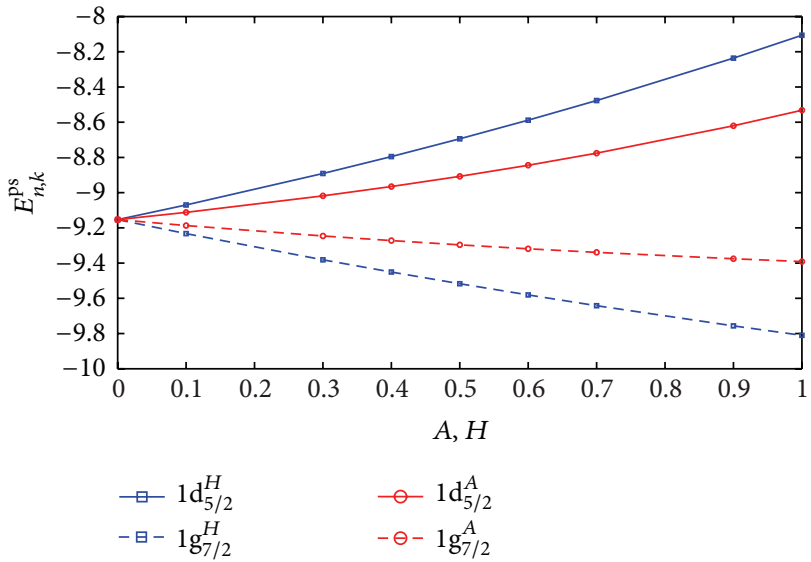

(b)

Figure 7: Energy spectra in the (a) spin and (b) pseudospin symmetries at various $H$ and $A$ for comparison between Coulomb and Yukawalike tensor interaction with parameters $M=10 \mathrm{fm}^{-1}, c=1, \hbar=1, \alpha=0.4 \mathrm{fm}^{-1}, V_{1}=4 \mathrm{fm}^{-1}, V_{0}=3 \mathrm{fm}^{-1}, q_{1}=1 \mathrm{fm}^{-1}, q_{2}^{\mathrm{ps}}=-2 \mathrm{fm}^{-1}$, $q_{2}^{s}=2 \mathrm{fm}^{-1}, C_{s}=5 \mathrm{fm}^{-1}$, and $C_{\mathrm{ps}}=-5 \mathrm{fm}^{-1}$.

we show the effect of Coulomb-like tensor interaction and Yukawa plus Coulomb-like tensor interaction in the remove of degeneracy.

In Figure 7, we show the comparison between Coulomb and Yukawa-like tensor interaction for spin and pseudospin symmetries.

In Figures 5, 6, and 7, we showed that degeneracy is removed by tensor interaction. Furthermore, the amount of the energy difference between the two states in the doublets increases with increasing $H$ and $A$.

\section{Results and Discussion}

We obtained the energy eigenvalues in the absence and the presence of the Coulomb-like tensor potential for various values of the quantum numbers $n$ and $k$. In Tables 1 and 4 , in the absence of the tensor interaction $(H=0)$, the degeneracy between spin doublets and pseudospin doublets is observed. For example, we observe the degeneracy in $\left(1 \mathrm{p}_{1 / 2}, 1 \mathrm{p}_{3 / 2}\right)$, $\left(1 d_{3 / 2}, 1 d_{5 / 2}\right)$, and so forth in the spin symmetry, and we observe the degeneracy in $\left(1 \mathrm{~s}_{1 / 2}, 1 \mathrm{~d}_{3 / 2}\right),\left(1 \mathrm{p}_{3 / 2}, 1 \mathrm{f}_{5 / 2}\right)$, and so forth in the pseudospin symmetry. When we consider the tensor interaction, for example, by parameter $H=0.65$, the degeneracy is removed. In Tables 2 and 3 for the spin symmetry and also in Tables 5 and 6 for the pseudospin symmetry, we show that degeneracy exists between spin doublets for several of the parameters $\alpha$ and $M$, and we show that degeneracy is removed in the presence of tensor interaction. In Figure 2, the degeneracy is removed by tensor interaction effect in spin symmetry and pseudospin symmetry; also the amount of the energy difference between the two states in the doublets increases with increasing parameter $H$. The effects of the Yukawa-like tensor interactions on the upper and lower components of radial Dirac equation for the symmetries are 
shown in Figures 3 and 4 . The sensitiveness of the pseudospin doublets $\left(1 \mathrm{p}_{3 / 2}, 1 \mathrm{f}_{5 / 2}\right)$ and $\left(1 \mathrm{~d}_{5 / 2}, 1 \mathrm{~g}_{7 / 2}\right)$ and spin doublets $\left(1 \mathrm{f}_{5 / 2}, 1 \mathrm{f}_{7 / 2}\right)$ and $\left(\mathrm{ld}_{3 / 2}, 1 \mathrm{~d}_{5 / 2}\right)$ for the effects of the Yukawalike tensor interactions and for Yukawa plus Coulomb-like tensor interaction is given in Figures 5 and 6. In Figure 7 we show that the Coulomb-tensor interaction is stronger than Yukawa-like tensor interaction to remove degeneracy.

\section{Conclusions}

In this paper, we have discussed approximately the solutions of the Dirac equation for Eckart plus Hulthen potentials with spin symmetry and pseudospin symmetry for $k \neq 0$. We obtained the energy eigenvalues and the wave function in terms of the generalized polynomials functions via the formula method. To show the accuracy of the present model, some numerical values of the energy levels are shown in Figures 3, 4, 5, and 6. We have showed that the energy degeneracy in pseudospin and spin doublets is removed by the tensor interaction effect.

\section{Conflict of Interests}

The authors declare that there is no conflict of interests regarding the publication of this paper.

\section{References}

[1] K. J. Oyewumi and C. O. Akoshile, "Bound-state solutions of the Dirac-Rosen-Morse potential with spin and pseudospin symmetry," The European Physical Journal A, vol. 45, no. 3, pp. 311-318, 2010.

[2] I. C. Wang and C. Y. Wong, "Finite-size effect in the Schwinger particle-production mechanism," Physical Review D, vol. 38, no. 1, pp. 348-359, 1988.

[3] P. Alberto, R. Lisboa, M. Malheiro, and A. S. de Castro, "Tensor coupling and pseudospin symmetry in nuclei," Physical Review C, vol. 71, no. 3, Article ID 034313, 2005.

[4] Y. I. Salamin, S. X. Hu, K. Z. Hatsagortsyan, and C. H. Keitel, "Relativistic high-power laser-matter interactions," Physics Reports, vol. 427, no. 2-3, pp. 41-155, 2006.

[5] M. I. Katsnelson, K. S. Novoselov, and A. K. Geim, "Chiral tunnelling and the Klein paradox in graphene," Nature Physics, vol. 2, no. 9, pp. 620-625, 2006.

[6] Y.-F. Cheng and T.-Q. Dai, "Exact solutions of the Klein-Gordon equation with a ring-shaped modified Kratzer potential," Chinese Journal of Physics, vol. 45, no. 5, pp. 480-487, 2007.

[7] A. Arima, M. Harvey, and K. Shimizu, "Pseudo LS coupling and pseudo SU3 coupling schemes," Physics Letters B, vol. 30, no. 8, pp. 517-522, 1969.

[8] K. T. Hecht and A. Adler, "Generalized seniority for favored $J \neq$ 0 pairs in mixed configurations," Nuclear Physics A, vol. 137, no. 1, pp. 129-143, 1969.

[9] J. N. Ginocchio, "Relativistic symmetries in nuclei and hadrons," Physics Reports, vol. 414, no. 4-5, pp. 165-261, 2005.

[10] R. Lisboa, M. Malheiro, A. S. de Castro, P. Alberto, and M. Fiolhais, "Pseudospin symmetry and the relativistic harmonic oscillator," Physical Review C, vol. 69, no. 2, Article ID 024319, 2004.
[11] M. Eshghi, "Makarov potential in relativistic equation via Laplace transformation approach," Canadian Journal of Physics, vol. 91, no. 1, pp. 71-74, 2013.

[12] O. Aydogdu and R. Sever, "Pseudospin and spin symmetry in the Dirac equation with Woods-Saxon potential and tensor potential," The European Physical Journal A, vol. 43, no. 1, pp. 73-81, 2010.

[13] H. Akcay and C. Tezcan, "Exact solutions of the Dirac equation with harmonic oscillator potential including a Coulomb-like tensor potential," International Journal of Modern Physics C, vol. 20, pp. 930-941, 2009.

[14] C.-S. Jia, P. Guo, and X.-L. Peng, "Exact solution of the DiracEckart problem with spin and pseudospin symmetry," Journal of Physics A: Mathematical and General, vol. 39, no. 24, p. 7737, 2006.

[15] A. Contreras-Astorga, D. J. Fernández, and J. Negro, "Solutions of the Dirac equation in a magnetic field and intertwining operators," Symmetry, Integrability and Geometry: Methods and Applications, vol. 8, article 082, 2012.

[16] H. Feizi, A. A. Rajabi, and M. R. Shojaei, "Supersymmetric solution of the Schrödinger equation for Woods-Saxon potential by using the Pekeris approximation," Acta Physica Polonica B, vol. 42, no. 10, pp. 2143-2152, 2011.

[17] H. Ciftci, R. L. Hall, and N. Saad, "Asymptotic iteration method for eigenvalue problems," Journal of Physics A: Mathematical and General, vol. 36, no. 47, pp. 11807-11816, 2003.

[18] O. Özer and G. Lévai, "Asymptotic iteration method applied to bound-state problems with unbroken and broken supersymmetry," Romanian Journal of Physics, vol. 57, no. 3-4, pp. 582-593, 2012.

[19] S.-H. Dong, Factorization Method in Quantum Mechanics, Springer, Dordrecht, The Netherlands, 2007.

[20] L. Infeld and T. E. Hull, "The factorization method," Reviews of Modern Physics, vol. 23, no. 1, pp. 21-68, 1951.

[21] A. Arda and R. Sever, "Exact solutions of the Morse-like potential, step-up and step-down operators via Laplace transform approach," Communications in Theoretical Physics, vol. 58, no. 1, pp. 27-30, 2012.

[22] A. K. Roy, "Calculation of the spiked harmonic oscillators through a generalized pseudospectral method," Physics Letters A, vol. 321, no. 4, pp. 231-238, 2004.

[23] A. K. Roy, "Studies on some exponential-screened Coulomb potentials," International Journal of Quantum Chemistry, vol. 113, no. 10, pp. 1503-1510, 2013.

[24] J. M. Cai, P. Y. Cai, and A. Inomata, "Path-integral treatment of the Hulthén potential," Physical Review A, vol. 34, no. 6, pp. 4621-4628, 1986.

[25] A. Diaf, A. Chouchaoui, and R. J. Lombard, "Feynman integral treatment of the Bargmann potential," Annals of Physics, vol. 317, no. 2, pp. 354-365, 2005.

[26] A. Diaf and A. Chouchaoui, "l-states of the Manning-Rosen potential with an improved approximate scheme and Feynman path integral formalism," Physica Scripta, vol. 84, no. 1, Article ID 015004, 2011.

[27] M. R. Shojaei, A. A. Rajabi, M. Farrokh, and N. Zoghi-Foumani, "Energy levels of spin-1/2 particles with Yukawa interaction," Journal of Modern Physics, vol. 5, no. 9, pp. 773-780, 2014.

[28] A. F. Nikiforov and V. B. Uvarov, Special Functions of Mathematical Physics, Birkhäuser, Berlin, Germany, 1988.

[29] M. R. Shojaei and M. Mousavi, "Solutions of the Klein-Gordon equation for $1 \neq 0$ with positiondependent mass for modified 
Eckart potential plus Hulthen potential," International Journal of Physical Sciences, vol. 10, no. 9, pp. 324-328, 2015.

[30] A. N. Ikot, A. B. Udoimuk, and L. E. Akpabio, "Bound states solution of Klein-Gordon equation with type-I equal vector and scalar Poschl-Teller potential for arbitray $l$-state," American Journal of Scientific and Industrial Research, vol. 2, no. 2, pp. 179183, 2011.

[31] W. C. Qiang, "Bound states of the Klein-Gordon equation for ring-shaped Kratzer-type potential," Chinese Physics, vol. 13, pp. 575-578, 2004

[32] W.-C. Qiang, "Bound states of the Klein-Gordon and Dirac equations for potential $V(r)=A \bar{r}^{2}-B \bar{r}^{1}$," Chinese Physics, vol. 12, no. 10, pp. 1054-1057, 2003.

[33] C. Berkdemir, A. Berkdemir, and R. Sever, "Systematical approach to the exact solution of the Dirac equation for a deformed form of the Woods-Saxon potential," Journal of Physics A: Mathematical and General, vol. 39, no. 43, pp. 1345513463, 2006.

[34] J.-Y. Guo and Z.-Q. Sheng, "Solution of the Dirac equation for the Woods-Saxon potential with spin and pseudospin symmetry," Physics Letters A, vol. 338, no. 2, pp. 90-96, 2005.

[35] X. C. Zhang, Q. W. Liu, C. S. Jia, and L. Z. Wang, "Bound states of the Dirac equation with vector and scalar Scarf-type potentials," Physics Letters A, vol. 340, no. 1-4, pp. 59-69, 2005.

[36] F. Scarf, "New soluble energy band problem," Physical Review, vol. 112, no. 4, pp. 1137-1140, 1958.

[37] C.-Y. Chen, "Exact solutions of the Dirac equation with scalar and vector Hartmann potentials," Physics Letters A, vol. 339, no. 3-5, pp. 283-287, 2005.

[38] A. de Souza Dutra and M. Hott, "Dirac equation exact solutions for generalized asymmetrical Hartmann potentials," Physics Letters A, vol. 356, no. 3, pp. 215-219, 2006.

[39] S. M. Ikhdair and B. J. Falaye, "Bound states of spatially dependent mass Dirac equation with the Eckart potential including Coulomb tensor interaction," European Physical Journal Plus, vol. 129, no. 1, pp. 1-15, 2014.

[40] A. D. Alhaidari, "Relativistic extension of shape-invariant potentials," Journal of Physics A: Mathematical and General, vol. 34, no. 46, pp. 9827-9833, 2001.

[41] M. Farrokh, M. R. Shojaeia, and A. A. Rajabi, "Klein-Gordon equation with Hulth'en potential and position-dependent mass," The European Physical Journal Plus, vol. 128, article 14, 2013.

[42] C. Eckart, "The penetration of a potential barrier by electrons," Physical Review, vol. 35, article 1303, 1930.

[43] B. J. Falaye, "Any $\ell$-state solutions of the Eckart potential via asymptotic iteration method," Central European Journal of Physics, vol. 10, pp. 960-965, 2012.

[44] B. J. Falaye, S. M. Ikhdair, and M. Hamzavi, "Formula method for bound state problems," Few-Body Systems, vol. 56, no. 1, pp. 63-78, 2015.

[45] W. Greiner, Relativistic Quantum Mechanics:Wave Equations, Springer, Berlin, Germany, 2000.

[46] G. R. Satchler, Direct Nuclear Reactions, Oxford University Press, London, UK, 1983.

[47] E. L. Hill, "The theory of vector spherical harmonics," American Journal of Physics, vol. 22, article 211, 1954.

[48] R. L. Greene and C. Aldrich, "Variational wave functions for a screened Coulomb potential," Physical Review A, vol. 14, no. 6, pp. 2363-2366, 1976.
[49] C.-S. Jia, T. Chen, and L.-G. Cui, "Approximate analytical solutions of the Dirac equation with the generalized PöschlTeller potential including the pseudo-centrifugal term," Physics Letters A, vol. 373, no. 18-19, pp. 1621-1626, 2009. 

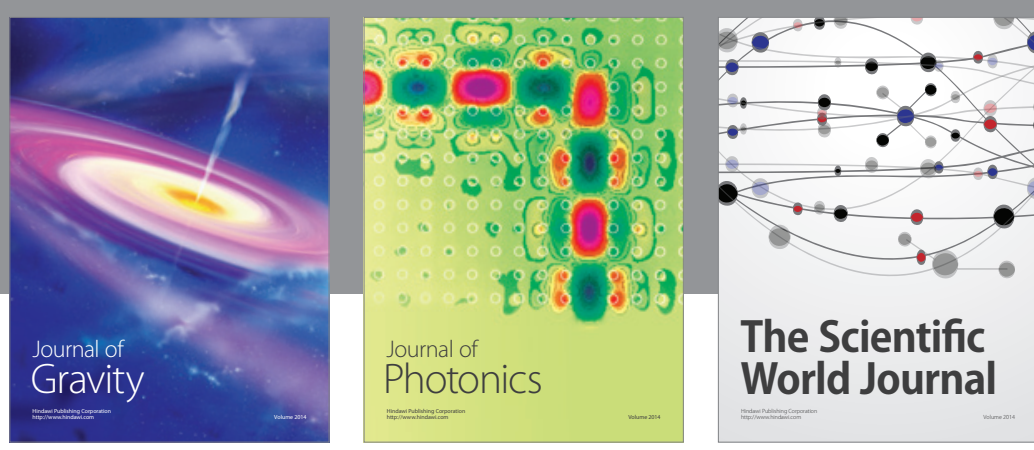

The Scientific World Journal
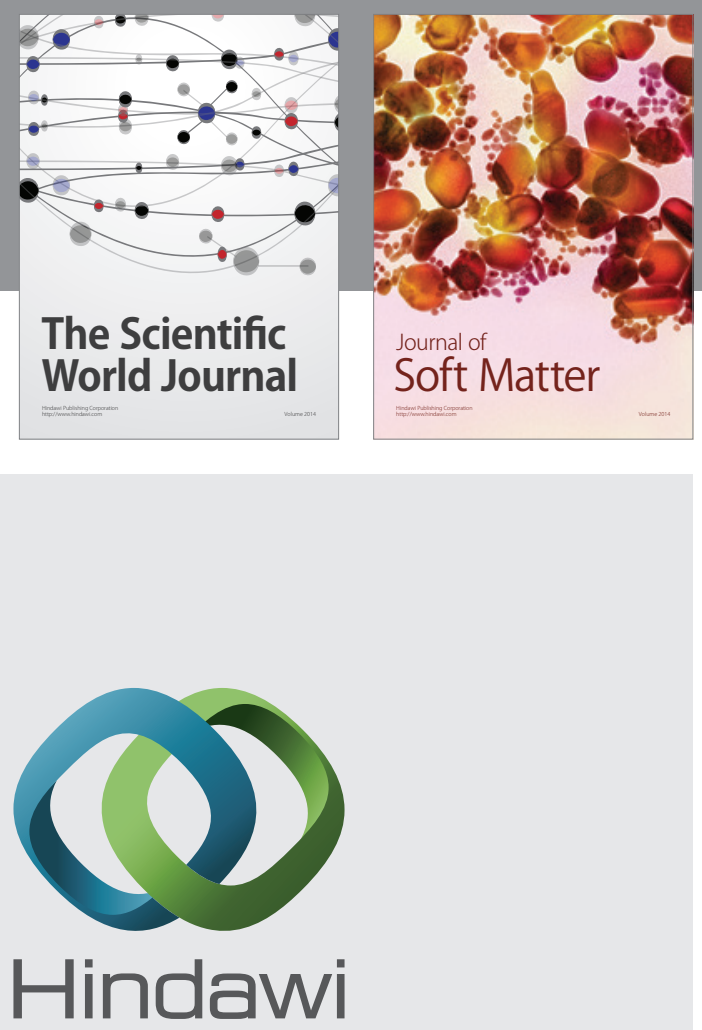

Submit your manuscripts at

http://www.hindawi.com

nternational Journal of

Statistical Mechanics
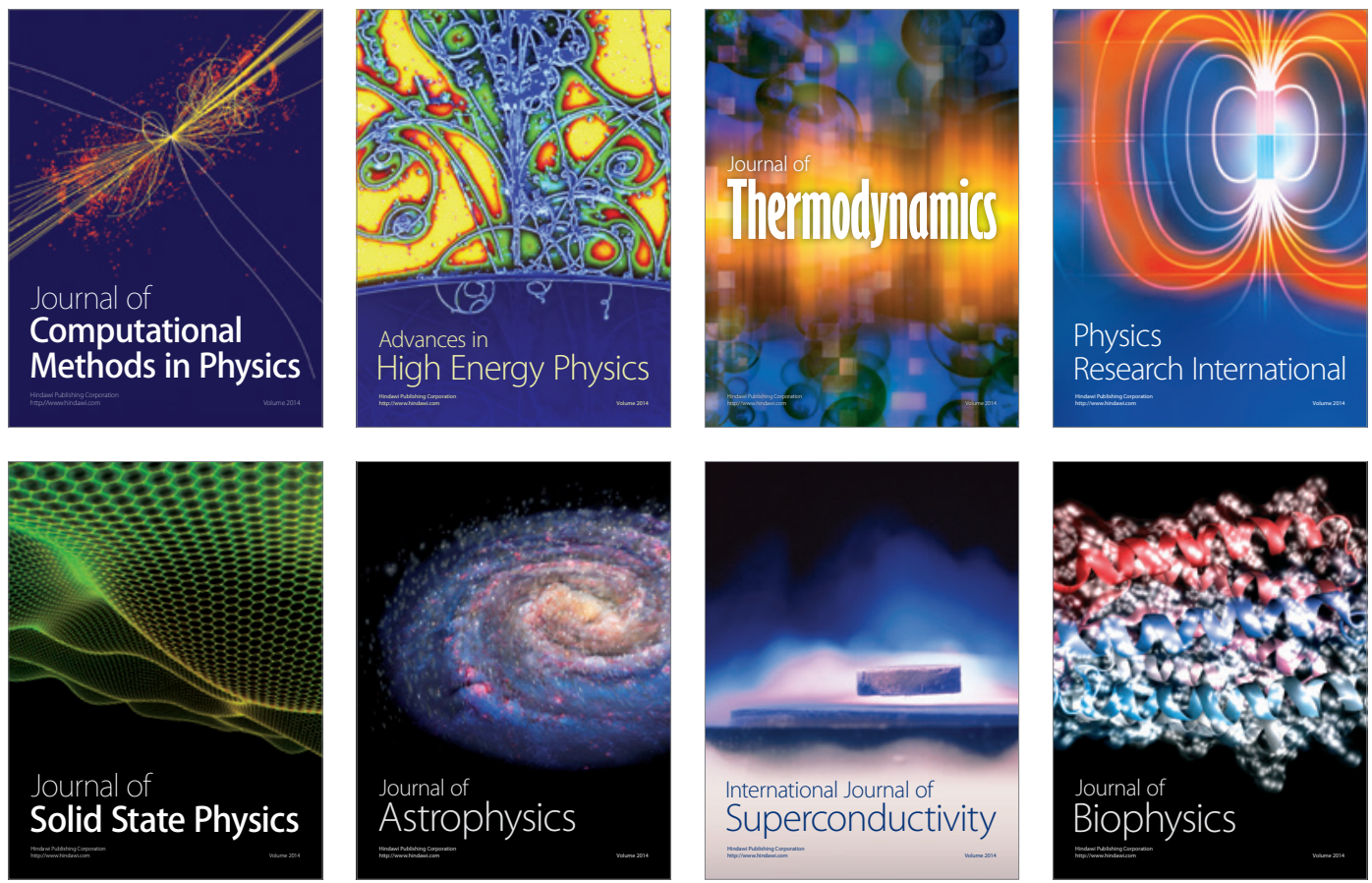
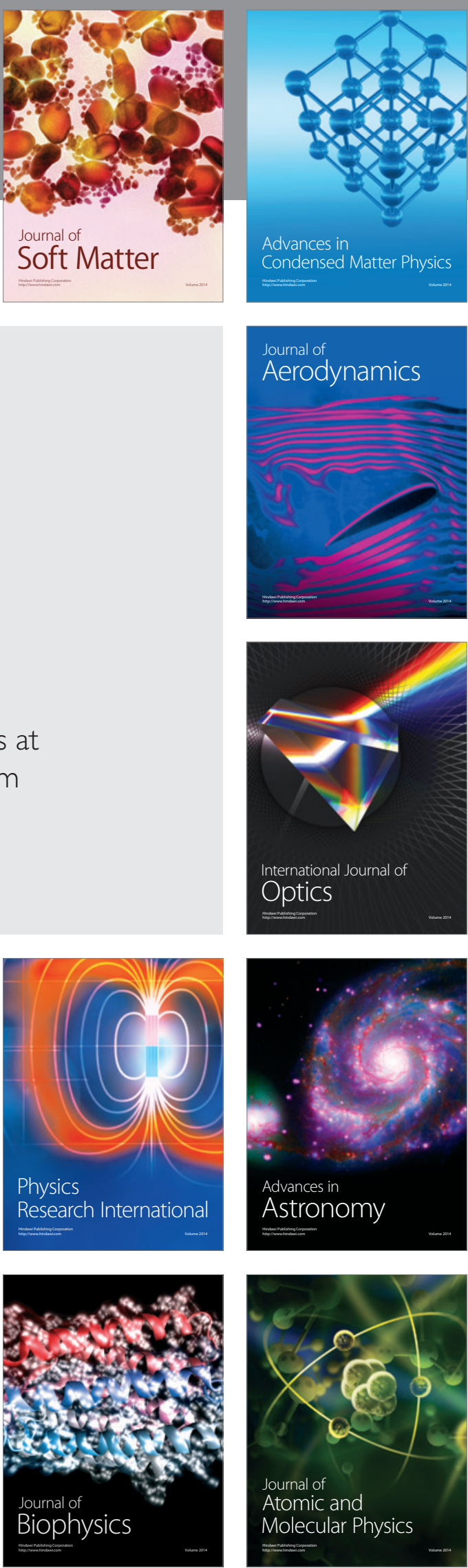